\title{
Vol. 68, No. 27
}

In the report "Vital Signs: Surveillance for Acute Flaccid Myelitis - United States, 2018," on page 609, in the first paragraph of the Results section, the fourth sentence should have read "Patients with illnesses classified as non-AFM were significantly older than were patients with confirmed AFM $($ median $=\mathbf{8 . 8}$ years $[$ range $=1$ month -78.1 years $] ; \mathrm{p}<0.001)$ (Table 1).”

On page 611, in Table 1, for "Laboratory finding; Spine MRI performed," the number of confirmed cases should have been "233/233 (100)," and the P-value should have been "0.10." In addition, for "Timing of preceding illness to onset of limb weakness, median days (range, IQR); Any respiratory illness," the interquartile range (IQR) for noncases should have been "6.5 (0-28, 4-11.5)." 